\title{
Comparison of OHAM and HPM methods for MHD flow of an upper-convected Maxwell fluid in a porous channel
}

R.Roslan $^{1, a}$, M.Abdulhameed ${ }^{1}$, B.S. Bhadauria ${ }^{2}$, and I. Hashim ${ }^{3}$

1 Faculty of Science, Technology and Human Development, Universiti Tun Hussein Onn, 86400 Batu Pahat, Johor, Malaysia

2 Department of Applied Mathematics, School of Physical Sciences, Babasaheb Bhimrao Ambedkar University, Lucknow, UP 226025, India

3 School of Mathematical Sciences, Universiti Kebangsaan Malaysia, 43600 Bangi Selangor, Malaysia

\begin{abstract}
This paper presents comparison between optimal homotopy asymptotic method and homotopy perturbation method for the solution of two-dimensional steady flow of an incompressible Maxwell fluid over a transpiration channel within a porous medium due to a impulsively moving wall. The results obtained using HPM for injection case fails to be in accordance with the available analytical data.
\end{abstract}

\section{Introduction}

Maxwell fluid is a subclass of non-Newtonian fluid of rate type with property to describe the relaxation time effects. Due to its ability in effectively taking various non-Newtonian effects, it has been the topic of several research Qi and $\mathrm{Xu}$ [2007], Hayat and Sajid [2007], Hayat et al. [2008], Jamil et al. [2011], Salah et al. [2011] and Hayat et al. [2011]. The governing equations are very complicated and highly nonlinear as compared to those for Newtonian fluids. There are few analytical solutions of the equations involving Newtonian fluids and such solutions become even rarer when equations for non-Newtonian fluids are considered. The aim of the present work is to solve the problem of the steady boundary layer flow of an upper-convected Maxwell fluid in a porous channel with wall transpiration. We have solved the governing nonlinear equation of present problem using the homotopy perturbation method, HPM, He [2005] and optimal homotopy asymptotic method, OHAM, Marinca and Herişanu [2008]. We noticed that the solution obtain from the two methods are in a very good agreement for suction case. It is noted from the solution series of homotopy perturbation method that the results in the case of injection fails to be in accordance with available analytical data.

\section{Statement of the problem}

Consider the steady MHD two-dimensional flow of an upperconvected Maxwell fluid in a channel with permeable walls. The distance between the channel width, is $2 l$ apart. Porous medium fills the space between the walls of the channel. The fluid motion is generated by suction/injection of the channel walls. The complete set of governing equations of the upper-convected Maxwell fluid consists of the incompressibility conditions

$$
\frac{\partial u}{\partial x}+\frac{\partial v}{\partial y}=0
$$

\footnotetext{
a e-mail: rozaini@uthm.edu.my
}

$$
\begin{aligned}
& u \frac{\partial u}{\partial x}+v \frac{\partial u}{\partial y}+\lambda\left[u^{2} \frac{\partial^{2} u}{\partial x^{2}}+v^{2} \frac{\partial^{2} u}{\partial y^{2}}+2 u v \frac{\partial^{2} u}{\partial x \partial y}\right] \\
= & v \frac{\partial^{2} u}{\partial y^{2}}-\frac{\sigma B_{0}^{2}}{\rho}\left(u+\lambda v \frac{\partial u}{\partial y}\right)-\frac{\phi v}{k} u,
\end{aligned}
$$

where $u$ and $v$ are the velocity components in the $x$ and $y$-axis, respectively, $\rho$ is the fluid density, $\lambda$ is the relaxation time, $k$ is the permeability of the porous medium, $\phi$ is the porosity, $v$ is the kinematic viscosity, $\sigma$ is the electrical conductivity and $B_{0}$ is the constant magnetic field in $y$ direction.

The appropriate boundary conditions are:

$$
\begin{aligned}
& u=0, v=\frac{V_{0}}{2}, \text { at } y= \pm \frac{l}{2}, \\
& \frac{\partial u}{\partial y}=0, v=0, \text { at } y=0,
\end{aligned}
$$

where $V_{0}$ is the transpiration velocity.

Hayat et al. [2011] has demonstrated that by using the similarity variables:

$$
\begin{gathered}
\tau=\frac{x}{l}, \eta=\frac{y}{l}, u=-V_{0} \tau \frac{d f(\eta)}{d \eta}, v=V_{0} f(\eta), \\
M=\frac{\sigma B_{0}^{2} l}{\rho V_{0}}, K=\frac{l^{2} \phi}{k}, \operatorname{Re}=\frac{l V_{0}}{v}, D e=\frac{\lambda V_{0}^{2}}{v} .
\end{gathered}
$$

The momentum equation (2) now can be reduced to the following similarity equation:

$$
\begin{array}{r}
\frac{d^{3} f}{d \eta^{3}}-M\left(\operatorname{Re} \frac{d f}{d \eta}+\operatorname{Def} \frac{d^{2} f}{d \eta}\right)-K \frac{d f}{d \eta}+\operatorname{Re}\left[\left(\frac{d f}{d \eta}\right)^{2}\right. \\
\left.-f \frac{d^{2} f}{d \eta^{2}}\right]+\operatorname{De}\left(2 f \frac{d f}{d \eta} \frac{d^{2} f}{d \eta^{2}}-f^{2} \frac{d^{3} f}{d \eta^{3}}\right)=0 .
\end{array}
$$

The corresponding boundary conditions (3) reduce to

$$
\begin{array}{ll}
f=\frac{1}{2}, & \frac{d f}{d \eta}=0, \\
f=0, & \frac{d^{2} f}{d \eta^{2}}=0, \quad \text { at } y= \pm \frac{1}{2},
\end{array}
$$


where $M$ is the Hartman number, $K$ is the permeability of the porous medium, $R e$ is the Reynolds number and $D e$ is the Deborah number. Must be noted that for $R e>0$ corresponds to suction case, $R e<0$ for injection and $D e=$ 0 for Newtonian fluid.

\section{Solution using the OHAM}

According to OHAM by Marinca and Herişanu [2008] the differential equation (5) satisfied by the velocity $f(\eta)$ is decomposed into a linear part $L(f)$ and a non-linear part $N(f)$ and is written in the form:

$$
L(f)+g(\eta)+N(f)=0, \quad B\left(f, \frac{d f}{d \eta}, \frac{d^{2} f}{d \eta^{2}}\right),
$$

where

$$
\begin{gathered}
L(f)=\frac{d^{3} f}{d \eta^{3}} \\
N(f)=-M\left(\operatorname{Re} \frac{d f}{d \eta}+\operatorname{Def} \frac{d^{2} f}{d \eta}\right)-K \frac{d f}{d \eta} \\
+\operatorname{Re}\left[\left(\frac{d f}{d \eta}\right)^{2}-f \frac{d^{2} f}{d \eta^{2}}\right]+D e\left(2 f \frac{d f}{d \eta} \frac{d^{2} f}{d \eta^{2}}-f^{2} \frac{d^{3} f}{d \eta^{3}}\right) .
\end{gathered}
$$

We now construct a homotopy $\psi(\eta, p): \mathbb{R} \times[0,1] \rightarrow \mathbb{R}$ which satisfies the family of equations:

$$
\begin{aligned}
&(1-p) \quad[L(\psi(\eta, p))+g(\eta)] \\
&=H(p)[L(\psi(\eta, p))+g(\eta)+N(\psi(\eta, p))], \\
& B\left(\psi(\eta, p), \frac{d \psi(\eta, p)}{\partial \eta}, \frac{d^{2} \psi(\eta, p)}{\partial \eta^{2}}\right)=0, \\
&(1-p) \quad \frac{d^{3} f(\eta, p)}{d \eta^{3}}=H(p)\left\{\frac{d^{3} f(\eta, p)}{d \eta^{3}}-M\left(\operatorname{Re} \frac{d f}{d \eta}\right.\right. \\
&\left.+D e f \frac{d^{2} f}{d \eta}\right)-K \frac{d f}{d \eta}+\operatorname{Re}\left[\left(\frac{d f}{d \eta}\right)^{2}-f \frac{d^{2} f}{d \eta^{2}}\right] \\
&\left.+D e\left(2 f \frac{d f}{d \eta} \frac{d^{2} f}{d \eta^{2}}-f^{2} \frac{d^{3} f}{d \eta^{3}}\right)\right\}, \\
& B\left(f(\eta, p), \frac{d f(\eta, p)}{\partial \eta}, \frac{d^{2} f(\eta, p)}{\partial \eta^{2}}\right)=0,
\end{aligned}
$$

where $p \in[0,1]$ is an embedding parameter. Expand $\psi(\eta, p)$ in a Taylor series with respect to $p$, we obtain

$\psi\left(\eta, p, C_{i}\right)=f_{0}(\eta)+\sum_{k=1}^{\infty} f_{k}\left(\eta, C_{i}\right) p^{k}, \quad$ and $i=1,2, \ldots, n$,

where $f_{0}(\eta)$ is the initial approximation. Using the boundary conditions (6), we choose the initial approximation $f_{0}(\eta)$ as

$$
f_{0}(\eta)=\frac{1}{2}\left(3 y-4 y^{3}\right)
$$

Substituting equation (12) into equation (11) and equating the coefficients of like powers of $p$, we obtain the following sets of problems for the first-order and second order problem respectively, as follows:

$$
\begin{aligned}
& \frac{d^{3} f_{1}}{d \eta^{3}}-\frac{d^{3} f_{0}}{d \eta^{3}}=C_{1}\left\{\frac{d^{3} f_{0}}{d \eta^{3}}-M\left(\operatorname{Re} \frac{d f_{0}}{d \eta}+D e f_{0} \frac{d^{2} f_{0}}{d \eta^{2}}\right)\right. \\
& -K \frac{d f_{0}}{d \eta}+\operatorname{Re}\left[\left(\frac{d f_{0}}{d \eta}\right)^{2}-f_{0} \frac{d^{2} f_{0}}{d \eta^{2}}\right] \\
& \left.+D e\left(2 f_{0} \frac{d f_{0}}{d \eta} \frac{d^{2} f_{0}}{d \eta^{2}}-f_{0}^{2} \frac{d^{3} f_{0}}{d \eta^{3}}\right)\right\}, \\
& f_{1}\left(\frac{1}{2}\right)=\frac{d f_{1}\left(\frac{1}{2}\right)}{d \eta}=f_{1}(0)=\frac{d^{2} f_{1}(0)}{d \eta^{2}}=0, \text { and }
\end{aligned}
$$

$$
\begin{aligned}
& \frac{d^{3} f_{2}}{d \eta^{3}}-\frac{d^{3} f_{1}}{d \eta^{3}}=C_{1}\left\{\frac{d^{3} f_{1}}{d \eta^{3}}-M\left[\operatorname{Re} \frac{d f_{1}}{d \eta}\right.\right. \\
& \left.+D e\left(f_{0} \frac{d^{2} f_{1}}{d \eta^{2}}+f_{1} \frac{d^{2} f_{0}}{d \eta^{2}}\right)\right]-K \frac{d f_{1}}{d \eta} \\
& +\operatorname{Re}\left[2 \frac{d f_{0}}{d \eta} \frac{d f_{1}}{d \eta}-\left(f_{0} \frac{d^{2} f_{1}}{d \eta^{2}}+f_{1} \frac{d^{2} f_{0}}{d \eta^{2}}\right)\right] \\
& +D e\left[2\left(f_{0} \frac{d f_{1}}{d \eta} \frac{d^{2} f_{1}}{d \eta^{2}}+f_{1} \frac{d f_{0}}{d \eta} \frac{d^{2} f_{1}}{d \eta^{2}}+f_{1} \frac{d f_{1}}{d \eta} \frac{d^{2} f_{0}}{d \eta^{2}}\right)\right. \\
& \left.\left.-\left(2 f_{0} \frac{d f_{0}}{d \eta} \frac{d f_{1}}{d \eta}+f_{0}^{2} \frac{d^{3} f_{1}}{d \eta^{3}}\right)\right]\right\} \\
& +C_{2}\left\{\frac{d^{3} f_{0}}{d \eta^{3}}-M\left(\operatorname{Re} \frac{d f_{0}}{d \eta}+D e f_{0} \frac{d^{2} f_{0}}{d \eta^{2}}\right)-K \frac{d f_{0}}{d \eta}\right. \\
& \left.+\operatorname{Re}\left[\left(\frac{d f_{0}}{d \eta}\right)^{2}-f_{0} \frac{d^{2} f_{0}}{d \eta^{2}}\right]+D e\left(2 f_{0} \frac{d f_{0}}{d \eta} \frac{d^{2} f_{0}}{d \eta^{2}}-f_{0}^{2} \frac{d^{3} f_{0}}{d \eta^{3}}\right)\right\}, \\
& f_{2}\left(\frac{1}{2}\right)=\frac{d f_{2}\left(\frac{1}{2}\right)}{d \eta}=f_{2}(0)=\frac{d^{2} f_{2}(0)}{d \eta^{2}}=0 .
\end{aligned}
$$

The second order approximate solution $\widetilde{f}$ :

$$
\widetilde{f}\left(\eta, C_{1}, C_{2}\right)=f_{0}+f_{1}+f_{2} .
$$

Hence, the approximate analytical solution (16) yields the following residual $R$ and the functional $J$, respectively

$$
\begin{gathered}
R(\eta)=\frac{d^{3} \widetilde{f}}{d \eta^{3}}-M\left(\operatorname{Re} \frac{d \widetilde{f}}{d \eta}+\operatorname{Def} \frac{d^{2} \widetilde{f}}{d \eta}\right)-K \frac{d \widetilde{f}}{d \eta} \\
+\operatorname{Re}\left[\left(\frac{d \widetilde{f}}{d \eta}\right)^{2}-\widetilde{f} \frac{d^{2} \widetilde{f}}{d \eta^{2}}\right]+D e\left(2 \widetilde{f} \frac{d \widetilde{f}}{d \eta} \frac{d^{2} \widetilde{f}}{d \eta^{2}}-\widetilde{f}^{2} \frac{d^{3} \widetilde{f}}{d \eta^{3}}\right) \\
J\left(C_{1}, C_{2}\right)=\int_{0}^{0.5} R^{2}\left(\eta, C_{1}, C_{2}\right) d \eta
\end{gathered}
$$

For calculation of constants $C_{1}$ and $C_{2}$ the method of least squares is employed.

The case of Newtonian fluid $(D e=0)$, by fixing $K=$ 0.5 and $M=1$, we obtained the approximate solutions of the second order for suction, $R e=20$ and injection, $R e=-20$ cases respectively as follows:

$$
\begin{aligned}
& f(\eta)=1.56123 \eta-2.62355 \eta^{3}+0.654338 \eta^{4}+0.382815 \eta^{6} \\
& -0.0203779 \eta^{7}+0.165849 \eta^{8}-0.0174164 \eta^{9} \\
& -0.00351136 \eta^{11}+0.00066536 \eta^{13}
\end{aligned}
$$


$f(\eta)=1.43713 \eta-1.35306 \eta^{3}-0.69448 \eta^{4}-0.336842 \eta^{6}$

$-0.0220769 \eta^{7}-0.14185 \eta^{8}-0.0175108 \eta^{9}$

$-0.00349076 \eta^{11}+0.00066536 \eta^{13}$.

While for the case of non-Newtonian fluid ( $D e=0.5$ ), by fixing $K=0.5$ and $M=1$, we obtained the approximate solutions of the second order for suction, $R e=20$ and injection, $R e=-20$ cases respectively as follows:

$$
\begin{aligned}
& f(\eta)=1.58804 \eta-3.01244 \eta^{3}+1.30439 \eta^{4} \\
& -0.037345 \eta^{5}+0.062715 \eta^{6}+0.000884155 \eta^{7} \\
& +0.327464 \eta^{8}-0.043228 \eta^{9}-0.0033277 \eta^{10} \\
& +0.0079121 \eta^{11}-0.00185403 \eta^{12}-0.0121708 \eta^{13} \\
& +0.000393708 \eta^{14}+0.000373307 \eta^{15}-0.000514279 \eta^{16} \\
& +0.0000150178 \eta^{18}-0.0000430097 \eta^{20}
\end{aligned}
$$

$$
\begin{aligned}
& f(\eta)=1.41147 \eta-0.993641 \eta^{3}-1.25752 \eta^{4} \\
& -0.0393603 \eta^{5}-0.0365252 \eta^{6}+0.0017511 \eta^{7} \\
& -0.275855 \eta^{8}-0.0403496 \eta^{9}+0.00645865 \eta^{10} \\
& +0.00403368 \eta^{11}+0.000642343 \eta^{12}-0.0116429 \eta^{13} \\
& -0.000189756 \eta^{14}-0.000390271 \eta^{15}+0.000376716 \eta^{16} \\
& -0.0000209551 \eta^{18}+0.0000430097 \eta^{20}
\end{aligned}
$$

\section{Solution using the HPM}

According to the homotopy perturbation method by $\mathrm{He}$ [2005], the differential equation (5) satisfied by the velocity field $f(\eta)$ is decomposed into a linear part $L(f)$ and a non-linear part $N(f)$ similar to (7)-(9). We construct a homotopy class $h(\eta, p): \mathbb{R} \times[0,1] \rightarrow \mathbb{R}$ which satisfies the following equation:

$$
L(h)-L\left(f_{0}\right)+p\left[L\left(f_{0}\right)+N(h)+g(\eta)\right]=0,
$$

where $f_{0}$ is an initial approximation to the solution $f(\eta)$. Assuming:

$$
f_{0}(\eta)=\frac{3 \eta}{2}-2 \eta^{3}
$$

Taking $p$ as small parameter and taking a power series solution of equation (26) in the form:

$$
h(\eta, p)=\sum_{k=0}^{\infty} p^{k} h_{k}(\eta),
$$

where $h_{k}$ are unknown function of $\eta$. Now setting $p \rightarrow 1$, equation (28) yield the approximate analytical solution of $f(\eta)$

$$
f(\eta)=\lim _{p \rightarrow 1} h(\eta, p)=\sum_{k=0}^{\infty} h_{k}(\eta) .
$$

Substituting equation (28) into equation (26) and the boundary conditions (6) and equate the coefficients of like powers of $p$ we obtain the following sets of problems for the first-order and second order problem respectively, as follows:

$$
\begin{aligned}
& \frac{d^{3} h_{1}}{d \eta^{3}}+\frac{d^{3} f_{0}}{d \eta^{3}}-M\left(\operatorname{Re} \frac{d h_{0}}{d \eta}+\operatorname{Deh}_{0} \frac{d^{2} h_{0}}{d \eta^{2}}\right)-K \frac{d h_{0}}{d \eta} \\
& +\operatorname{Re}\left[\left(\frac{d h_{0}}{d \eta}\right)^{2}-h_{0} \frac{d^{2} h_{0}}{d \eta^{2}}\right] \\
& +D e\left(2 h_{0} \frac{d h_{0}}{d \eta} \frac{d^{2} h_{0}}{d \eta^{2}}-h_{0}^{2} \frac{d^{3} h_{0}}{d \eta^{3}}\right)=0, \\
& h_{1}\left(\frac{1}{2}\right)=\frac{d h_{1}\left(\frac{1}{2}\right)}{d \eta}=h_{1}(0)=\frac{d^{2} h_{1}(0)}{d \eta^{2}}=0, \text { and }(27) \\
& \frac{d^{3} h_{2}}{d \eta^{3}}-M\left[\operatorname{Re} \frac{d h_{1}}{d \eta}+D e\left(h_{0} \frac{d^{2} h_{1}}{d \eta^{2}}+h_{1} \frac{d^{2} h_{0}}{d \eta^{2}}\right)\right]-K \frac{d h_{1}}{d \eta} \\
& +\operatorname{Re}\left[2 \frac{d h_{0}}{d \eta} \frac{d h_{1}}{d \eta}-\left(h_{0} \frac{d^{2} h_{1}}{d \eta^{2}}+h_{1} \frac{d^{2} h_{0}}{d \eta^{2}}\right)\right] \\
& +D e\left[2\left(h_{0} \frac{d h_{1}}{d \eta} \frac{d^{2} h_{1}}{d \eta^{2}}+h_{1} \frac{d h_{0}}{d \eta} \frac{d^{2} h_{1}}{d \eta^{2}}+h_{1} \frac{d h_{1}}{d \eta} \frac{d^{2} h_{0}}{d \eta^{2}}\right)\right. \\
& \left.-\left(2 h_{0} \frac{d h_{0}}{d \eta} \frac{d h_{1}}{d \eta}+h_{0}^{2} \frac{d^{3} h_{1}}{d \eta^{3}}\right)\right]=0 \\
& h_{2}\left(\frac{1}{2}\right)=\frac{d h_{2}\left(\frac{1}{2}\right)}{d \eta}=h_{2}(0)=\frac{d^{2} h_{2}(0)}{d \eta^{2}}=0 .
\end{aligned}
$$

In view of equation (29), the second order approximate solution is given in the form

$$
f(\eta)=h_{0}(\eta)+h_{1}(\eta)+h_{2}(\eta) .
$$

The case of Newtonian fluid $(D e=0)$, by fixing $K=$ 0.5 and $M=1$, we obtained the approximate solutions of the second order for suction, $R e=20$ and injection, $R e=-20$ cases respectively as follows:

$$
\begin{aligned}
f(\eta)= & 1.44998 \eta-1.50564 \eta^{3}-0.501723 \eta^{4}-0.244683 \eta^{6} \\
& -0.142783 \eta^{7}-0.061756 \eta^{8}-0.122032 \eta^{9} \\
& -0.0246032 \eta^{11}+0.004662 \eta^{13} \\
f(\eta)= & 1.56525 \eta-2.68744 \eta^{3}+0.75588 \eta^{4}+0.424023 \eta^{6} \\
& -0.154688 \eta^{7}+0.229911 \eta^{8}-0.122693 \eta^{9} \\
& -0.0244589 \eta^{11}+0.004662 \eta^{13}
\end{aligned}
$$

The case of Newtonian fluid $(D e=0.5)$, by fixing $K=$ 0.5 and $M=1$, we obtained the approximate solutions of the second order for suction, $R e=20$ and injection, $R e=-20$ cases respectively as follows:

$$
\begin{aligned}
& f(\eta)=1.42938 \eta-1.25824 \eta^{3}-0.765788 \eta^{4} \\
& -0.261667 \eta^{5}-0.308506 \eta^{6}+0.000695406 \eta^{7} \\
& -0.109331 \eta^{8}-0.239972 \eta^{9}+0.00497605 \eta^{10} \\
& +0.00015907 \eta^{11}+0.017362 \eta^{12}-0.0790405 \eta^{13} \\
& -0.00417921 \eta^{14}-0.00692374 \eta^{15}+0.00741998 \eta^{16} \\
& -0.000278536 \eta^{18}+0.000797702 \eta^{20}
\end{aligned}
$$

$$
\begin{aligned}
& f(\eta)=1.59395 \eta-3.14132 \eta^{3}+1.62956 \eta^{4} \\
& -0.275788 \eta^{5}+0.0711154 \eta^{6}+0.0182914 \eta^{7}+0.47375 \eta^{8} \\
& -0.352074 \eta^{9}+0.0183066 \eta^{10} \\
& +0.0900576 \eta^{11}-0.0302276 \eta^{12}-0.0888314 \eta^{13} \\
& +0.00682448 \eta^{14}+0.00723836 \eta^{15}-0.00910533 \eta^{16} \\
& +0.000388655 \eta^{18}-0.000797702 \eta^{20}
\end{aligned}
$$


(a)

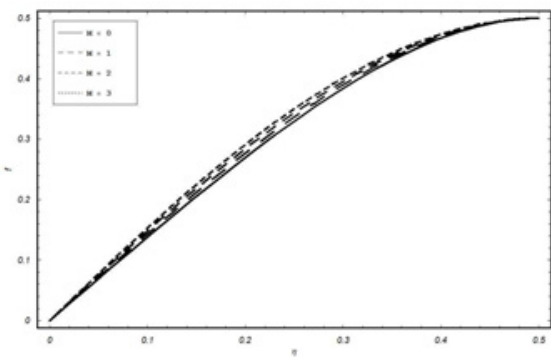

(b)

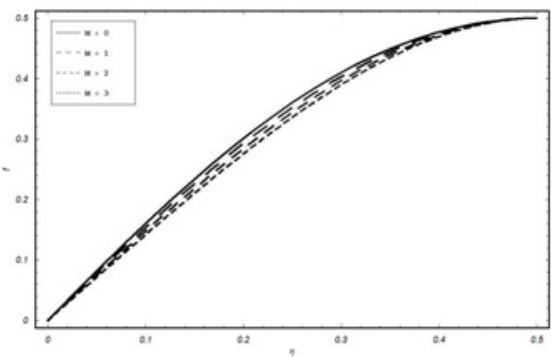

Fig. 1. Effects of Hartman number $M$ on $f$ in the case of suction $R e>0$ (a) and injection $R e<0$ (b) using HPM

\section{Analysis of result}

The results for Newtonian fluid $(D e=0)$ are demonstrated in Fig. 1 and 2. Fig. 1 illustrate the influence of Hartman number $M$, on the non-dimensional velocity using HPM for suction case (Fig. $1(a)$ ) and injection (Fig. $1(b)$ ). It is observed that for suction and injection $f$ shows reverse opposite behavior when $M$ varies. This behavior is within contrast using the available analytical data (Hayat et al. [2011] Fig. 4 and Fig. 5). The influence of Hartman number $M$, on the non-dimensional velocity using OHAM for suction case (Fig. $2(a)$ ) and injection (Fig. $2(b)$ ). is shown in Fig. 2. It is observed that for suction and injection $f$ shows good agreement using the available analytical data (Hayat et al. [2011]. As $M$ increases the velocity decreases in the boundary layer region for injection case (Fig. $2(b)$ ). This is physically true because the application of magnetic field to an electrically conducting fluid gives rise to resistive force which is known as Lorentz force. When suction is present in (Fig. $2(a)$ ), we should expect the reverse effect since the momentum transmitted to the fluid by the wall is sucked away. While, the results for nonNewtonian fluid $(D e \neq 0)$ are demonstrated in Fig. 3 and 4. Fig. 3 illustrate the variation of Hartman number $H$ on the non-dimensional velocity $f$ using HPM with suction case (Fig. $3(a)$ ) and injection (Fig. $3(b)$ ). The result show similar behaviour as that for Newtonian fluid (Fig. 1) and also the results are contrast with the available analytical data (Hayat et al. [2011] Fig. 16 and Fig. 22). Fig. 4 shows the variation of Hartman number $H$ on the non-dimensional velocity $f$ using OHAM with suction case (Fig. $4(a)$ ) and injection (Fig. $4(b)$ ). The result show similar behaviour as that for Newtonian fluid (Fig. 2) and also the results are in good agrement with the available analytical data (Hayat et al. [2011])

\section{Conclusion remark}

Approximate analytical solutions for velocity upper-convected Maxwell fluid in a porous channel with wall transpiration

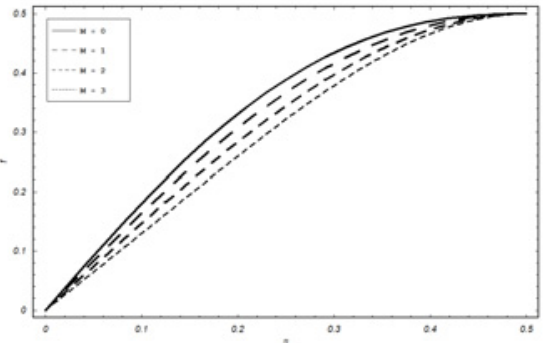

(a)

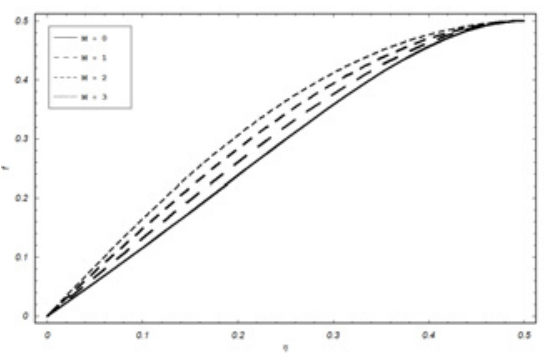

(b)

Fig. 2. Effects of Hartman number $M$ on $f$ in the case of suction $R e>0$ (a) and injection $R e<0$ (b) using OHAM

(a)

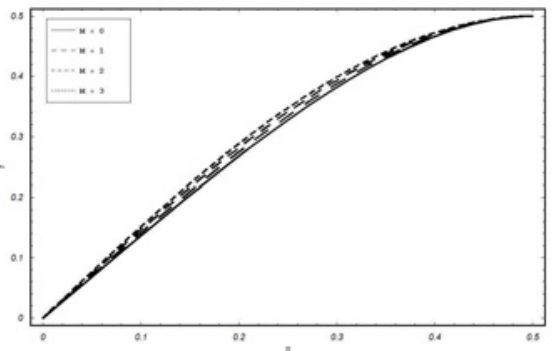

(b)

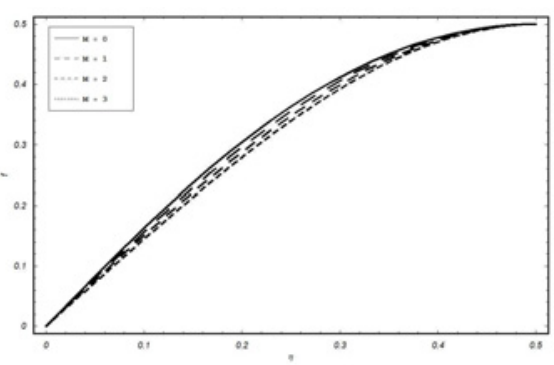

Fig. 3. Effects of Hartman number $M$ on $f$ in the case of suction $R e>0$ (a) and injection $R e<0$ (b) using HPM

have been discovered. The governing nonlinear differential equations are solved using both the Homotopy perturbation (HPM) method and Optimal homotopy asymptotic method (OHAM) and the results are validated by comparing the present paper with the results of the related published articles. The process using OHAM is found explicit, effective and it has a definite edge on homotopy perturbation method for the reason that the approximate solution acquired could be modified good convergence and low error. The results obtained using HPM for injection case fails to be in accor- 


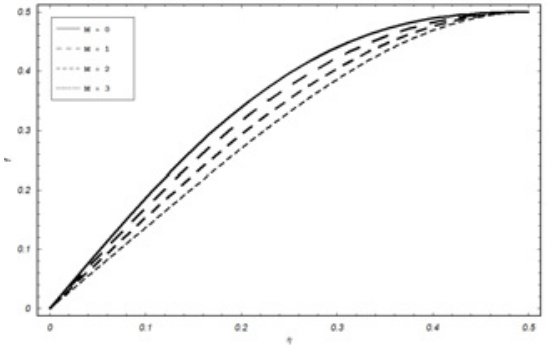

(a)

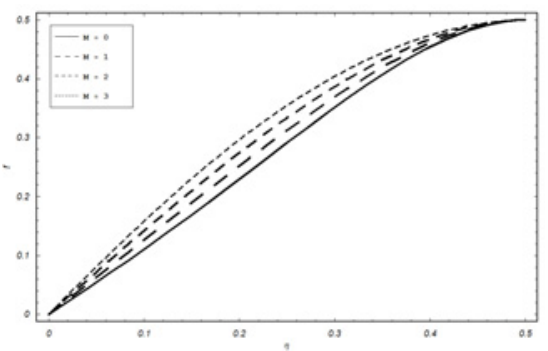

(b)

Fig. 4. Effects of Hartman number $M$ on $f$ in the case of suction $R e>0$ (a) and injection $R e<0$ (b) using OHAM

dance with the available analytical data.

\section{Acknowledgments}

The author would like to acknowledge the financial support received from the Grant FRGS/2/2010/SG

/UTHM/02/4/0761.

\section{References}

T.Hayat, R.Sajjad, Z.Abbas, M.Sajid, A.H.Awatif, Radiation effects on MHD flow of Maxwell fluid in a channel with porous medium, International Journal of Heat and Mass Transfer. 54 (2011) 854-862.

T.Hayat, C.Fetecau, Z.Abbas, N.Ali, Flow of a Maxwell fluid between two side walls due to a suddenly moved plate, Nonlinear Analysis: Real World Applications. 9(5) (2008) 2288-2295.

T,Hayat, M.Sajid, Homotopy analysis of MHD boundary layer flow of an upper-convected Maxwell fluid, International Journal of Engineering Science. 45 (2007) 393401.

M,Jamil, A.Rauf, A.A.Zafar, N.A.Khan, New exact analytical solutions for Stokes' first problem of Maxwell fluid with fractional derivative approach, Computers and Mathematics with Applications. 62(3) (2011) 10131023.

H.Qi, M.Xu, Unsteady flow of viscoelastic fluid with fractional Maxwell model in a channel, Mechanics Research Communications. 34(2) (2007) 210-212.

F.Salah, Z.A.Aziz, D.L.C.Ching, New exact solution for Rayleigh-Stokes problem of Maxwell fluid in a porous medium and rotating frame, Results in Physics. 1(1) (2011) 9-12.
J.H.He, Application of homotopy perturbation method to nonlinear wave equations, Chaos, Solitons and Fractals. 26(3) (2005) 695-700.

V.Marinca, N.Herişanu, Application of Optimal Homotopy Asymptotic Method for solving nonlinear equations arising in heat transfer, International Communications in Heat and Mass Transfer. 35(6) (2008) 710-715. 